ARTICLE OPEN

\title{
Chromosomal microarray analysis of 410 Han Chinese patients with autism spectrum disorder or unexplained intellectual disability and developmental delay
}

Yi Liu (iD ${ }^{1,6}$, Yuqiang Lv ${ }^{1,6}$, Mehdi Zarrei ${ }^{2}$, Rui Dong ${ }^{1}$, Xiaomeng Yang ${ }^{1}$, Edward J. Higginbotham $\mathbb{D}^{2}$, Yue $\mathrm{Li}^{1}$, Dongmei Zhao ${ }^{3}$, Fengling Song ${ }^{3}$, Yali Yang ${ }^{4}$, Haiyan Zhang ${ }^{1}$, Ying Wang ${ }^{1}$, Stephen W. Scherer $\mathbb{D}^{2,5 凶}$ and Zhongtao Gai ${ }^{1 凶}$

Copy number variants (CNVs) are recognized as a crucial genetic cause of neurodevelopmental disorders (NDDs). Chromosomal microarray analysis (CMA), the first-tier diagnostic test for individuals with NDDs, has been utilized to detect CNVs in clinical practice, but most reports are still from populations of European ancestry. To contribute more worldwide clinical genomics data, we investigated the genetic etiology of 410 Han Chinese patients with NDDs (151 with autism and 259 with unexplained intellectual disability (ID) and developmental delay (DD)) using CMA (Affymetrix) after G-banding karyotyping. Among all the NDD patients, 109 (26.6\%) carried clinically relevant CNVs or uniparental disomies (UPDs), and 8 (2.0\%) had aneuploidies (6 with trisomy 21 syndrome, 1 with 47,XXY, 1 with 47,XYY). In total, we found 129 clinically relevant CNVs and UPDs, including 32 CNVs in 30 ASD patients, and 92 CNVs and 5 UPDs in 79 ID/DD cases. When excluding the eight patients with aneuploidies, the diagnostic yield of pathogenic and likely pathogenic CNVs and UPDs was 20.9\% for all NDDs (84/402), 3.3\% in ASD (5/151), and 31.5\% in ID/DD (79/251). When aneuploidies were included, the diagnostic yield increased to $22.4 \%$ for all NDDs (92/410), and 33.6\% for ID/DD (87/259). We identified a de novo CNV in 14.9\% (60/402) of subjects with NDDs. Interestingly, a higher diagnostic yield was observed in females $(31.3 \%, 40 / 128)$ compared to males $(16.1 \%, 44 / 274)$ for all NDDs $\left(P=4.8 \times 10^{-4}\right)$, suggesting that a female protective mechanism exists for deleterious CNVs and UPDs.

npj Genomic Medicine (2022)7:1 ; https://doi.org/10.1038/s41525-021-00271-z

\section{INTRODUCTION}

Neurodevelopmental disorders (NDDs) are a group of heterogeneous disorders involving developmental dysfunction of the central nervous system, and have an incidence rate of $1-3 \%$ in children ${ }^{1-3}$. Autism spectrum disorder (ASD) and intellectual disability/developmental delay (ID/DD) are the most common NDDs in children and have shared psychiatric behaviors, clinical manifestations, and risk factors, and impair cognitive functions including learning, sociability, and mood ${ }^{4}$. ASD is one of the childhood-onset NDDs, characterized by impairment in three domains: social interaction, communication skills, and repetitive behavior and restricted interests. Approximately $50 \%$ of autistic cases manifest intellectual disability (ID) ${ }^{5,6}$. Developmental delay (DD) is the failure to achieve certain developmental milestones at the appropriate age, involving physical, cognitive, communication, social, emotional, and/or adaptive skills ${ }^{3}$. Both ID $(I Q<70)$ and $D D$ belong to the clinically heterogeneous NDDs ${ }^{3}$.

The etiology of ASD and ID/DD is complex and overlapping, implicating both genetic and non-genetic factors. Chromosomal microarray analysis (CMA) is a molecular cytogenetic technique that expedites genome-wide detection of clinically significant copy number variants (CNVs), and has been recommended as a first-tier diagnostic tool for patients with ASD, unexplained ID/DD, and multiple congenital anomalies (MCA). CMA can accurately detect different types of CNVs (i.e., deletions or duplications) across a large size range (esp. $<5 \mathrm{Mb}$ submicroscopic CNVs), and can also identify uniparental disomies (UPDs) ${ }^{7-9}$. CMA has been demonstrated to improve the diagnostic yield to up to $33 \%$, compared to $3.7 \%$ using karyotyping. However, the diagnostic yield can vary widely and largely depends on the cohort population and severity of phenotypes ${ }^{9-11}$.

CNV studies have been performed for patients with NDDs worldwide, but additional data from Chinese subjects are needed to delineate the potential differences in CNV distribution in this population, particularly within the clinical setting. Here, we present an investigation of CNVs in a well-characterized clinical cohort with ASD or ID/DD in Shandong, a province in northern China. CNVs were analyzed using the Affymetrix SNP 6.0 or CytoScan HD arrays in combination with deep mining databases, including an updated in-house database, to explore the clinical implication and inheritance of specific candidate CNVs or genes associated with NDDs.

\section{RESULTS}

\section{General diagnostic yield}

Samples from 410 Han Chinese ASD and ID/DD patients were tested, of which 5 with highly suspected trisomy 21 syndrome were analyzed by G-banding karyotyping. Trisomy 21 was detected in these five samples. The remaining 405 samples were analyzed by array and three additional aneuploidies were found: one trisomy 21 , one $47, X X Y$, and one $47, X Y Y$. Thus, aneuploidies were identified in eight subjects (2.0\%) in our cohort, with six trisomy 21 , one $47, X X Y$, and one $47, X Y Y$ identified.

\footnotetext{
'Pediatric Research Institute, Qilu Children's Hospital of Shandong University, Ji'nan 250022, China. ${ }^{2}$ The Centre for Applied Genomics and Department of Genetics and Genome Biology, The Hospital for Sick Children, Toronto, ON M5G 0A4, Canada. ${ }^{3}$ Pediatric Health Care Institute, Qilu Children's Hospital of Shandong University, Ji'nan 250022, China. ${ }^{4}$ Rehabilitation Center, Qilu Children's Hospital of Shandong University, Ji'nan 250022, China. ${ }^{5}$ McLaughlin Centre and Department of Molecular Genetics, University of Toronto, Toronto, ON M5S 1A1, Canada. ${ }^{6}$ These authors contributed equally: Yi Liu, Yuqiang Lv. ${ }^{凶}$ email: stephen.scherer@sickkids.ca; gaizhongtao@sina.com
} 
Table 1. Diagnostic yield of CNVs identified in the cohort.

\begin{tabular}{|c|c|c|c|c|c|c|c|}
\hline $\begin{array}{l}\text { Clinical } \\
\text { diagnosis }\end{array}$ & $\begin{array}{l}\text { Age } \\
\text { (years) }\end{array}$ & Total no. & Sex, no. & $\begin{array}{l}\text { Chromosomal } \\
\text { syndrome (No.) }\end{array}$ & Pathogenic (No.) & Likely pathogenic (No.) & $\begin{array}{l}\text { Diagnostic } \\
\text { yield (\%) }\end{array}$ \\
\hline \multirow[t]{7}{*}{ ASD } & $1-2$ & 2 & Male, 2 & 0 & 0 & 0 & 0 \\
\hline & \multirow[t]{2}{*}{$2-5$} & \multirow[t]{2}{*}{114} & Male, 94 & 3 & 3 & 0 & $4.3(3 / 94)$ \\
\hline & & & Female, 20 & 1 & 1 & 0 & $5.0(1 / 20)$ \\
\hline & $>5$ & 35 & Male, 31 & 0 & 0 & 1 & $2.9(1 / 35)$ \\
\hline & \multirow[t]{3}{*}{ Total } & \multirow[t]{3}{*}{151} & Male, 127 & 3 & 3 & 1 & $3.1(4 / 127)$ \\
\hline & & & Female, 24 & 1 & 1 & 0 & $4.2(1 / 24)$ \\
\hline & & & $M+F, 151$ & 4 & 4 & 1 & $3.3(5 / 151)$ \\
\hline \multirow[t]{7}{*}{ ID/DD } & \multirow[t]{2}{*}{$<1$} & \multirow[t]{2}{*}{104} & Male, 58 & 11 & 13 & 0 & $22.4(13 / 58)$ \\
\hline & & & Female, 46 & 7 & 10 & 3 & $28.3(13 / 46)$ \\
\hline & \multirow[t]{2}{*}{$>5$} & \multirow[t]{2}{*}{17} & Male, 13 & 0 & 1 & 0 & $7.7(1 / 13)$ \\
\hline & & & Female, 4 & 2 & 2 & 0 & $50.0(2 / 4)$ \\
\hline & \multirow[t]{3}{*}{ Total } & \multirow[t]{3}{*}{251} & Male, 147 & 33 & 39 & 1 & $27.2(40 / 147)$ \\
\hline & & & Female, 104 & 29 & 36 & 3 & $37.5(39 / 104)$ \\
\hline & & & $M+F, 251$ & 62 & 75 & 4 & $31.5(79 / 251)$ \\
\hline \multirow[t]{3}{*}{ ASD + ID/DD } & \multirow[t]{3}{*}{ Total } & \multirow[t]{3}{*}{402} & Male, 274 & 35 & 42 & 2 & $16.1(44 / 274)$ \\
\hline & & & Female, 128 & 29 & 37 & 3 & $31.3(40 / 128)$ \\
\hline & & & $M+F, 402$ & 64 & 79 & 5 & $20.9(84 / 402)$ \\
\hline
\end{tabular}

The remaining 402 cases ( 151 ASD and 251 ID/DD) were further analyzed for the presence of clinically relevant CNVs, which were defined as pathogenic CNVs, likely pathogenic CNVs, and CNVs interpreted as variants of uncertain significance (VUS). We detected 594 high-quality CNVs in our cohort (Supplementary Data 2), and selected 162 rare CNVs for experimental validation, of which 150/162 were confirmed as true calls. We interpreted 129 CNVs and uniparental disomies (UPDs) as clinically relevant, which were identified in 109 NDD cases (27.1\%, 109/402). This included 32 CNVs in 30 ASD cases (19.9\%), and 97 CNVs and UPDs in 79 ID/DD cases (31.5\%). Four loci of mosaicism were found in our cohort: 9p24 duplication (x3-4), 15q11.2q13.3 duplication (x3-4), 18q21.31q23 deletion (x1-2), and 22q11.1q11.21 duplication $(x 3-4)$.

The 32 clinically relevant CNVs identified in 30 ASD cases included four pathogenic CNVs associated with known genetic syndromes, one likely pathogenic CNV, and 27 CNVs interpreted as variants of uncertain significant (VUS) according to ACMG classification (Table 2). There were $14(43.8 \%)$ clinically relevant deletions and 18 (56.3\%) clinically relevant duplications identified, of which five (15.6\%) occurred de novo, 25 (78.1\%) were inherited (16 paternal and 9 maternal), and two (6.3\%) were of unknown inheritance (Tables 1 and 2, and Supplementary Data 3).

In contrast, the 97 CNVs and UPDs in ID/DD cases included 90 pathogenic variants (72 implicating 34 known genetic syndromes), four likely pathogenic variants, and three VUS based on ACMG classification (Tables 1 and 3). The clinically relevant CNVs and UPDs included 53 (54.6\%) deletions, 39 (40.2\%) duplications, and five $(5.2 \%)$ UPDs. The majority of CNVs $(67.01 \%, 65 / 97)$ were de novo, and the proportion of ID/DD subjects with a de novo CNV was $21.9 \%(55 / 251)$, much higher than that of ASD subjects $(P=$ $\left.1.0 \times 10^{-7}\right)$. We found that $12.4 \%$ of the CNVs and UPDs were inherited ( 3 paternal and 9 maternal), and $20.6 \%$ were of unknown inheritance due to the unavailability of parental samples (Table 1 and 3, and Supplementary Data 4). The inheritance of the five UPDs was determined, revealing two cases with Prader-Willi syndrome, one case with Angelman syndrome, one case with Silver-Russell syndrome, and one case with maternal UPD in 14q.

To obtain a more stringent estimate of the diagnostic yield for our cohort, we considered only subjects with pathogenic/likely pathogenic CNVs and UPDs in the calculation. The diagnostic yield was $20.9 \%$ (84/402) for all NDDs, and increased to $22.4 \%(92 / 410)$ when subjects with aneuploidies were included. The diagnostic yield was markedly lower for ASD $(5 / 151,3.3 \%)$ compared to ID/ DD (79/251, 31.5\% without aneuploidies; 87/259, 33.6\% including aneuploidies).

The size range of the clinically relevant CNVs and UPDs was $56 \mathrm{~kb}$ to $95.1 \mathrm{Mb}$ in the entire cohort, $56 \mathrm{~kb}$ to $8.4 \mathrm{Mb}$ in subgroup of ASD cases, and $377 \mathrm{~kb}$ to $95.1 \mathrm{Mb}$ in the subgroup of ID/DD cases. The average size of positive CNVs and UPDs was $802.5 \mathrm{~kb} \pm$ $394.5 \mathrm{~kb}$ in ASD cases compared to $4.14 \mathrm{Mb} \pm 3.77 \mathrm{Mb}$ in ID/DD cases, which showed a significant difference between the two subgroups $(P=0.005)$. We then compared the average size of CNVs and UPDs identified in males and females of both subgroups. There was no statistical difference in the average size of CNVs identified in males and females in the ASD subgroup (802.5 kb $\pm 394.5 \mathrm{~kb}$ in males compared to $316 \mathrm{~kb} \pm 201 \mathrm{~kb}$ in females). In contrast, the average size of CNVs and UPDs was $2.45 \mathrm{Mb} \pm 2.07 \mathrm{Mb}$ in males and $20.12 \mathrm{Mb} \pm 12.21 \mathrm{Mb}$ in females in the ID/DD subgroup, indicating a significant difference between male and female ID/DD cases $(P=0.001)$. The majority of clinically relevant CNVs and UPDs (95/129, 73.6\%) were smaller than $10 \mathrm{Mb}$ in size (i.e., submicroscopic) and would not be identified by karyotyping (Tables 2 and 3 and Supplementary Data 4). 


\begin{tabular}{|c|c|c|c|c|c|c|c|}
\hline $\begin{array}{l}\text { CADM2 }+ \\
10 \text { genes }\end{array}$ & 1 & $3 p 12.2 p 11.1$ & 8418 & Deletion & De novo & - & Likely pathogenic \\
\hline AUTS2 & 1 & $7 q 11.22$ & 830 & Deletion & De novo & AUTS2 syndrome & Pathogenic \\
\hline $\begin{array}{l}\text { BBS4, NEO1 }+ \\
31 \text { genes }\end{array}$ & 1 & $15 q 24.1 q 24.2$ & 2581 & Deletion & De novo & $\begin{array}{l}15 q 24 \text { microdeletion } \\
\text { syndrome }\end{array}$ & Pathogenic \\
\hline$M E C P 2+15$ genes & 1 & $\mathrm{Xq} 28$ & 408 & Duplication & Maternal & $\begin{array}{l}\text { MECP2 microduplication } \\
\text { syndrome }\end{array}$ & Pathogenic \\
\hline \multicolumn{8}{|c|}{ Variants of uncertain significance (VUS) } \\
\hline CTNNA2 +6 genes & 1 & $2 \mathrm{p} 12$ & 1197 & Duplication & Paternal & - & VUS \\
\hline GRM7 & 2 & $3 p 26.1$ & $303-1174$ & Deletion & $\begin{array}{l}\text { De novo (1), } \\
\text { maternal (1) }\end{array}$ & - & VUS \\
\hline$R S R C 1$ & 1 & $3 q 25.32$ & 435 & Deletion & Paternal & - & VUS \\
\hline PIGG & 1 & $4 p 16.3$ & 159 & Deletion & Paternal & - & VUS \\
\hline $\begin{array}{l}\text { ARHGEF38 }+ \\
3 \text { genes }\end{array}$ & 1 & $4 q 24$ & 162 & Duplication & Paternal & - & VUS \\
\hline KHDRBS2 & 1 & $6 q 12$ & 699 & Duplication & Paternal & - & VUS \\
\hline AHI1 & 1 & $6 q 23.3$ & 103 & Duplication & Paternal & - & VUS \\
\hline PARK2 & 2 & $6 q 26$ & $76-174$ & Deletion & Paternal (2) & - & VUS \\
\hline SDK1 & 1 & $7 p 22.2$ & 119 & Duplication & Unknown & - & VUS \\
\hline GRM8 & 1 & $7 q 31.33$ & 56 & Duplication & Paternal & - & VUS \\
\hline$L A M A 1+2$ genes & 1 & $18 p 11.23 p 11.31$ & 517 & Duplication & Unknown & - & VUS \\
\hline MIB1 & 1 & $18 q 11.2$ & 122 & Deletion & Maternal & - & VUS \\
\hline PTPRT & 1 & $20 q 12$ & 77 & Deletion & Maternal & - & VUS \\
\hline AlFM3 & 1 & $22 q 11.21$ & 412 & Duplication & Paternal & - & VUS \\
\hline IGBP1 + 10 genes & 1 & $\mathrm{Xq13.1}$ & 401 & Duplication & Maternal & - & VUS \\
\hline$S M S+P H E X$ & 1 & Xq22.11 & 75 & Duplication & Maternal & - & VUS \\
\hline
\end{tabular}

\section{CNVs in regions of known chromosomal syndromes}

Seventy-six CNVs were identified at regions associated with 37 well-known chromosomal microdeletion/microduplication syndromes. Four pathogenic CNVs associated with known genetic syndromes were identified in four ASD cases. A 4-year-old boy with ASD and DD harbored an $830 \mathrm{~kb}$ deletion at $7 \mathrm{q} 11.22$ that overlapped the AUTS2 gene. He was diagnosed with AUTS2 syndrome. A 3-year-6-month girl with ASD, DD and congenital heart disease harbored a $9 q 34.3$ deletion and was diagnosed with Kleefstra syndrome. A 2-year-6-month boy with ASD and DD harbored a $2.58 \mathrm{Mb}$ deletion at chr15q24, and was considered to have 15 q24 microdeletion syndrome. Last, a 3-year-3-month male patient harbored a $408 \mathrm{~kb}$ duplication at chrXq28 including $M E C P 2$, and was diagnosed with MECP2 duplication syndrome (Table 2 and Supplementary Data 3).

There were $73 \mathrm{CNV}$ s and four UPDs identified in 60 ID/DD cases that were associated with known chromosomal syndromes. Williams-Beuren syndrome deletions were the most commonly observed syndromic CNV, and were identified in 11 cases (Table 3). Examples of other CNVs and UPDs identified in multiple cases with ID/DD included five partial trisomy $9 p$, four $15 q 11 q 13$ duplication syndrome, four $18 q$ deletion syndrome, four Prader-Willi syndrome ( 2 paternal deletions and 2 maternal UPDs), and three 22q11 deletion syndrome, among others (Table 3 and Supplementary Data 4).

The patients with known chromosomal syndromes presented with heterogeneous clinical features, including three cases with de novo deletions of the $22 q 11.2$ deletion syndrome region. The first 22q11.2 deletion syndrome case, 15D1529, was a 23-month-15day-old boy, the first child of non-consanguineous healthy parents. His clinical features included special appearance such as sparse hairs, small eyes, low-ears, thick lips, and irregular teeth. He had a height of $83.5 \mathrm{~cm}$, weight of $10 \mathrm{~kg}$, and head circumference of $45.1 \mathrm{~cm}$. His speech was delayed significantly 
Y. Liu et al.

Table 3. Clinically relevant CNVs found in the patients with intellectual disability and developmental delay.

\begin{tabular}{|c|c|c|c|c|c|c|}
\hline Region name & $\begin{array}{l}\text { No. of } \\
\text { subjects }\end{array}$ & Cytoband & CNV type & Size (kb) & Parent of origin & Pathogenicity \\
\hline \multicolumn{7}{|l|}{ Genetic syndromes } \\
\hline $\begin{array}{l}\text { 1q21.1 microduplication } \\
\text { syndrome }\end{array}$ & 1 & $1 \mathrm{q} 21.1 \mathrm{q} 21.2$ & Duplication & 1626 & Maternal & Pathogenic \\
\hline Wolf-Hirschhorn syndrome & 1 & $4 p 16.3 p 16.2$ & Deletion & 5868 & De novo & Pathogenic \\
\hline Cri-du-chat syndrome & 2 & $\begin{array}{l}\text { 5p15.33p15.1; } \\
5 p 15.31 \text { p15.1 }\end{array}$ & Deletion & $7595-16,576$ & De novo (2) & Pathogenic \\
\hline $5 q$ trisomy syndrome & 1 & $5 q 34 q 35.3$ & Duplication & 15,248 & De novo & Pathogenic \\
\hline Sotos syndrome & 1 & $5 q 35.2 q 35.3$ & Deletion & 1911 & De novo & Pathogenic \\
\hline $\begin{array}{l}\text { Branchio-Otorenal } \\
\text { syndrome }\end{array}$ & 1 & $8 q 12.3 q 13.3$ & Deletion & 10,009 & De novo & Pathogenic \\
\hline $\begin{array}{l}9 \text { partial monosomy } \\
\text { syndrome }\end{array}$ & 1 & $9 p 24.3 p 23$ & Deletion & 10,487 & De novo & Pathogenic \\
\hline $9 p$ partial trisomy & 5 & $\begin{array}{l}\text { 9p24.3p13.1; } \\
\text { 9p24.3p21.1; } \\
\text { 9p24.3q21.11; } \\
\text { 9p24.3p21.13 }\end{array}$ & $\begin{array}{l}\text { Duplication (4), duplication } \\
x 3+\text { duplication } x 4(1)^{\mathrm{a}}\end{array}$ & $29,995-74,596$ & De novo (5) & Pathogenic \\
\hline 10p partial deletion & 1 & 10p15.3p14 & Deletion & 11,049 & De novo & Pathogenic \\
\hline 10p15.3 deletion syndrome & 1 & $10 p 15.3 p 15.2$ & Deletion & 3472 & De novo & Pathogenic \\
\hline $\begin{array}{l}\text { Distal trisomy 10q } \\
\text { syndrome }\end{array}$ & 1 & $10 q 24.2 q 26.3$ & Duplication & 35,938 & De novo & Pathogenic \\
\hline $\begin{array}{l}15 q 11-q 13 \text { duplication } \\
\text { syndrome }\end{array}$ & 4 & $\begin{array}{l}15 q 11.2 q 13.1 \\
15 q 11.2 q 13.2 \\
15 q 13.2 q 13.3\end{array}$ & $\begin{array}{l}\text { Duplication (1), duplication } \\
\text { x3 (1), duplication } \times 4(1) \text {, } \\
\text { duplication } \times 3+ \\
\text { duplication } \times 4(1)^{\mathrm{b}}\end{array}$ & $4929-9674$ & $\begin{array}{l}\text { De novo (3), } \\
\text { unknown (1) }\end{array}$ & Pathogenic \\
\hline $15 q 26$ deletion syndrome & 1 & $15 q 26.3$ & Deletion & 3134 & Unknown & Pathogenic \\
\hline $\begin{array}{l}\text { 16p13.11 microduplication } \\
\text { syndrome }\end{array}$ & 1 & $16 p 13.11$ & Duplication & 1429 & maternal & VUS \\
\hline $\begin{array}{l}\text { 16p11.2 microduplication } \\
\text { syndrome (BP4-BP5) }\end{array}$ & 2 & $16 p 11.2$ & Duplication & $586-826$ & $\begin{array}{l}\text { De novo (1), } \\
\text { unknown (1) }\end{array}$ & Pathogenic \\
\hline $\begin{array}{l}16 \text { partial trisomy } \\
\text { syndrome }\end{array}$ & 1 & $16 q 22.1 q 24.3$ & Duplication & 19,668 & De novo & Pathogenic \\
\hline $\begin{array}{l}17 \text { partial trisomy } \\
\text { syndrome }\end{array}$ & 1 & $17 q 25.3$ & Duplication & 5701 & De novo (1) & Pathogenic \\
\hline $18 q$ deletion syndrome & 4 & $\begin{array}{l}18 q 21.2 q 21.31 \\
18 q 21.31 q 23 \\
18 q 21.33 q 23 \\
18 q 22.3 q 23\end{array}$ & $\begin{array}{l}\text { Deletion (3), deletion } \\
\text { x1-2 (1) }\end{array}$ & $3042-77,943$ & $\begin{array}{l}\text { De novo (3), } \\
\text { unknown (1) }\end{array}$ & Pathogenic \\
\hline 18q duplication syndrome & 1 & $18 q 21.32 q 23$ & Duplication & 77,943 & De novo & Pathogenic \\
\hline $\begin{array}{l}22 q 11.2 \text { duplication } \\
\text { syndrome }\end{array}$ & 1 & $22 q 11.1 q 11.21 ; 22 q 11.21$ & $\begin{array}{l}\text { Duplication } \times 4+ \\
\text { duplication } \times 3^{c}\end{array}$ & $2893 ; 1682$ & De novo & Pathogenic \\
\hline $22 q 11.2$ deletion syndrome & 3 & $22 q 11.21$ & Deletion & 2549-2884 & De novo (3) & Pathogenic \\
\hline $\begin{array}{l}\text { MECP2 microduplication } \\
\text { syndrome }\end{array}$ & 2 & Xq28 & Duplication & $377-424$ & $\begin{array}{l}\text { Maternal (1), } \\
\text { paternal (1) }\end{array}$ & Pathogenic \\
\hline
\end{tabular}




\begin{tabular}{|c|c|c|c|c|c|c|}
\hline Region name & $\begin{array}{l}\text { No. of } \\
\text { subjects }\end{array}$ & Cytoband & CNV type & Size $(k b)$ & Parent of origin & Pathogenicity \\
\hline 1q25.1q31.1 deletion & 1 & 1q25.1q31.1 & Deletion & 12,292 & De novo & Pathogenic \\
\hline 2p16.1p12 deletion & 1 & $2 \mathrm{p} 16.1 \mathrm{p} 12$ & Deletion & 16,664 & Unknown & Pathogenic \\
\hline 2p21p13.2 duplication & 1 & $2 p 21 p 13.2$ & Duplication & 28,560 & De novo & Pathogenic \\
\hline $2 q 13$ deletion & 1 & $2 q 13$ & Deletion & 1734 & De novo & $\begin{array}{l}\text { Likely } \\
\text { pathogenic }\end{array}$ \\
\hline 3p14.1p11.1 duplication & 1 & 3p14.1p11.1 & Duplication & 21,577 & De novo & Pathogenic \\
\hline 4p16.3p16.1 duplication & 1 & $4 p 16.3 p 16.1$ & Duplication & 9452 & Unknown & Pathogenic \\
\hline $4 p 16.3$ deletion & 1 & $4 p 16.3$ & Deletion & 1195 & Unknown & Pathogenic \\
\hline 4p16.2p16.1 duplication & 1 & $4 p 16.2 p 16.1$ & Duplication & 493 & De novo & VUS \\
\hline $7 q 11.22 q 21.11$ deletion & 1 & $7 q 11.22 q 21.11$ & Deletion & 17,988 & De novo & $\begin{array}{l}\text { Likely } \\
\text { pathogenic }\end{array}$ \\
\hline 8p23.3p22 deletion & 1 & $8 p 23.3 p 22$ & Deletion & 17,300 & De novo & Pathogenic \\
\hline 13q21.1q32.2 deletion & 1 & $13 q 21.1 q 32.2$ & Deletion & 39,073 & De novo & Pathogenic \\
\hline 14q11.2q21.2 duplication & 1 & $14 q 11.2 q 21.2$ & Duplication & 23,595 & Unknown & Pathogenic \\
\hline $14 q 11.2 q 32.33$ UPD & 1 & $14 q 11.2 q 32.33$ & UPD & 86,774 & maternal & Pathogenic \\
\hline $\begin{array}{l}14 q 32.12 p 32.33 \\
\text { duplication }\end{array}$ & 1 & $14 q 32.12 p 32.33$ & Duplication & 11,616 & Unknown & Pathogenic \\
\hline $15 q 26.2 q 26.3$ deletion & 1 & $15 q 26.2 q 26.3$ & Deletion & 3006 & De novo & Pathogenic \\
\hline 16q24.2q24.3 duplication & 1 & $16 q 24.2 q 24.3$ & Duplication & 2671 & Unknown & Pathogenic \\
\hline 18p11.32p11.31 deletion & 1 & 18p11.32p11.31 & Deletion & 5987 & De novo & Pathogenic \\
\hline $21 q 22.11 q 22.3$ deletion & 1 & $21 q 22.11 q 22.3$ & Deletion & 13,055 & De novo & Pathogenic \\
\hline 21q22.3 duplication & 1 & $21 q 22.3$ & Duplication & 2858 & De novo & VUS \\
\hline $\begin{array}{l}\text { 22q13.31q13.33 } \\
\text { duplication }\end{array}$ & 2 & $22 q 13.31 q 13.33$ & Duplication & 5648 & Unknown (2) & Pathogenic \\
\hline Xq22.3q27.1 duplication & 1 & $X q 22.3 q 27.1$ & Duplication & 32,324 & Unknown & Pathogenic \\
\hline
\end{tabular}

with only babbling. His development was assessed using the Gesell Developmental Observation-Revised (GDO-R), and his motor, social, and language development were all found to be delayed. His blood biochemical tests, head MRI and heart ultrasound were normal. The second case, 15D3173, was an 8-day-old boy, who was the third child of non-consanguineous healthy parents, and had two healthy sisters of 10 years old and 7 years old, respectively. He was referred to the hospital because of seizures and abnormal development, and presented with facial dysmorphism, specifically small mouth, micrognathia, and high arched palate. His growth delay in utero was found before he was born, and physical examination and ultrasonic cardiogram demonstrated that he had atrioventricular septal defect (AVSD), and pulmonary arterial hypertension. His blood tests showed he had hypocalcemia with calcium $1.38 \mathrm{mmol} / \mathrm{L}$ and immunodeficiency with $\operatorname{lgA} 0.04 \mathrm{~g} / \mathrm{L}$ (normal: $0.03-0.82 \mathrm{~g} / \mathrm{L}$ ), $\operatorname{lgG} 8.37 \mathrm{~g} / \mathrm{L}$ (normal: $7.00-14.40 \mathrm{~g} / \mathrm{L}$ ), lgM $0.052 \mathrm{~g} / \mathrm{L}$ (normal: $0.06-0.20 \mathrm{~g} / \mathrm{L}$ ). His cryptorchidism and polydactyl were also noticed. The third case, 19D0970, was a 6-day-old girl and the first child of nonconsanguineous healthy parents. She was referred to the hospital for seizures and fever, and presented with facial dysmorphism, with features that included small jaw, flat bridge of nose, narrow nasal passages, laryngeal dysplasia with softening of the laryngeal cartilage, and softening of the trachea. She also had growth delay in utero before her birth. Ultrasound examination showed she suffered from congenital heart defects of patent ductus arteriosus, 
pulmonary hypertension, tricuspid regurgitation, and patent foramen ovala as well as smaller thymus $\left(1.7^{*} 1.5^{*} 0.6 \mathrm{~cm}\right)$. Her blood tests showed she had lower calcium $(0.83 \mathrm{mmol} / \mathrm{L}$, normal: 2.2-3.0 mmol/L) and congenital hypothyroidism with TSH $15.22 \mathrm{mlU} / \mathrm{L}$ (normal: $0.98-5.63 \mathrm{mlU} / \mathrm{L}$ ) and FT4 $2.53 \mathrm{pmol} / \mathrm{L}$ (normal: 11.4-19.5 pmol/L).

\section{De novo variants}

We identified 70 de novo CNVs in 60 patients with NDDs. This included 65 de novo CNVs identified in 55 ID/DD cases $(31$ males and 24 females) and five de novo CNVs identified in five ASD cases (4 males and 1 female). The proportion of subjects with de novo CNVs was $14.9 \%(60 / 402)$ for all 402 NDD patients (excludes patients with aneuploidies), 3.3\% (5/151) for ASD cases and $21.9 \%$ $(55 / 251)$ for the ID/DD cases. The proportion of subjects with a de novo CNVs was significantly higher in the ID/DD subgroup compared to the ASD subgroup $\left(P=1.0 \times 10^{-7}\right)$ (Tables 2 and 3, and Supplementary Data $3 \& 4$ ).

Of the 70 de novo CNVs found in NDDs, $75.7 \%$ (53/70) occurred at loci associated with known chromosomal syndromes, including three CNVs identified in ASD cases and 50 CNVs identified in ID/DD cases. The three syndromic de novo CNVs in ASD subjects included AUTS2 syndrome deletion, Kleefstra syndrome deletion, and $15 q 24$ microdeletion syndrome (Table 2). The remaining two de novo CNVs identified in two ASD cases were an 8.4 Mb deletion at $3 p 12.2 \mathrm{p} 11.1$ and a $303 \mathrm{~kb}$ deletion at 3p26.1. The de novo deletion at $3 p 12.2 p 11.1$ was detected in a 5-year-6-month boy who was diagnosed with severe autism. The deletion overlapped several genes that are highly expressed in the brain and might contribute to the phenotype, such as CADM2, CHMP2B, POU1F1, and CGGBP1. The other de novo deletion at 3 p26.1 was found in a 4-year-old boy, and overlapped GRM7, the gene of metabotropic glutamate receptor 7 that is an emerging candidate gene for ASD and other neuropsychiatric disorders ${ }^{12,13}$.

Of the 65 de novo CNVs found in ID/DD cases, 50 were associated with known chromosomal syndromes (Table 3). Notably, the deletions of 7q11.23 Williams-Beuren syndrome region occurred de novo in all 11 patients, who were diagnosed with Williams-Beuren syndrome. Other examples of de novo CNVs associated with genetic syndromes included five de novo duplications of 9p24 (partial trisomy 5p), three de novo duplications of 15q11-13, and two subjects de novo deletions of 2q37. There were 15 de novo CNVs that occurred at regions that were not associated with known chromosomal syndromes. These included 10 pathogenic CNVs, three likely pathogenic CNVs, and two CNVs classified as VUS based on ACMG guideline (Table 3 and Supplementary Data 4).

\section{Cases with multiple clinically relevant CNVs}

There were 20 cases who harbored two clinically relevant CNVs, of which two were ASD cases and 18 were ID/DD cases (Supplementary Data 3 and 4). The first ASD case (F3) was a 2-year-4month-old girl who harbored a $115 \mathrm{~kb}$ maternally transmitted duplication overlapping TRIP12 and a $74 \mathrm{~kb}$ maternally transmitted deletion overlapping CTNNA3, both of which were interpreted as VUS. The second ASD case (Y26) was a 3-year-10-month-old girl who harbored two CNVs interpreted as VUS: a $699 \mathrm{~kb}$ paternally transmitted duplication overlapping KHDRBS2 and a $76 \mathrm{~kb}$ paternally transmitted deletion overlapping PARK2. The 18 ID/DD cases included 15 cases with two pathogenic (or likely pathogenic) CNVs, and three subjects with one pathogenic CNV and a second CNV interpreted as VUS. For example, a 1-year-old girl (16D1511) with DD and facial abnormalities harbored both a $6.5 \mathrm{Mb}$ duplication at $2 \mathrm{p} 25.3 \mathrm{p} 25.2$ and a $23.6 \mathrm{Mb}$ duplication at chr14q11.2-q21.2. A 1-month-22-day-old girl (19D0185) with DD, bilateral hearing problem, valgus feet, and cleft palate had both a 17.38 $\mathrm{Mb}$ deletion at chr18q21.33q23 and a $9.84 \mathrm{Mb}$ deletion at chr20p13p12.3. A 9.45 Mb duplication at chr4p16.3p16.1 and a 6.84 Mb deletion at chr8p23.3p23.1 were detected in a 19-month8-day-old boy (19D1091) with DD, speech delay, and hearing problem. We found two cases with Williams-Beuren syndrome deletions who harbored a second clinically relevant CNV. Case (16D2191) harbored a de novo Williams-Beuren syndrome deletion and carried an additional pathogenic CNV, a $5.65 \mathrm{Mb}$ duplication at 22q13.31q13.33. The second case (19D0262) harbored a Williams-Beuren syndrome deletion and a recurrent 1.4 Mb duplication at 16p13.11, which was interpreted as VUS ${ }^{14}$.

\section{New CNV candidates potentially related to ASD}

In addition to the five pathogenic and likely pathogenic CNVs identified in the ASD subgroup, we identified 27 additional VUS in these cases (Table 2 and Supplementary Data 3). The VUS included 10 deletions and 17 duplications. One CNV occurred de novo, 24 were inherited, and two were of unknown inheritance. Analysis of data from the literature and disease databases suggests that many of these VUS are novel candidate CNVs for ASD and involve genes related to ASD/DD. Examples include a $303 \mathrm{~kb}$ de novo deletion and a $1.1 \mathrm{Mb}$ maternal deletion at 3p26.1 that both overlap GRM7; a $141 \mathrm{~kb}$ paternal duplication at chr2q14.1 overlapping DPP10; a $477 \mathrm{~kb}$ maternal duplication at chr2q14.3 overlapping CNTNAP5; a $115 \mathrm{~kb}$ maternal duplication at chr2q36.3 overlapping TRIP12; a $435 \mathrm{~kb}$ paternal deletion at chr3q25.32 overlapping $R S R C 1$; a $56 \mathrm{~kb}$ paternal duplication at chr7q31.33 overlapping GRM8; and a $452 \mathrm{~kb}$ paternal duplication at chr16p13.2 overlapping GRIN2A, among others (Supplementary Data 3).

\section{DISCUSSION}

The diagnostic yield of CMA varies across NDDs, with a higher detection rate of CNVs in patients with ID/DD than those with simplex ASD ${ }^{15-22}$. CMA has been applied for Chinese patients with NDDs in recent years, but the potential differences in CNV distribution in a clinical setting in China is not well illuminated. Here, we investigated the genetic etiology of 410 patients with ASD or ID/DD who were referred to our institute for clinical service by first using G-banding karyotyping and then genotyping samples using the Affymetrix SNP array 6.0 or CytoScan HD. Both platforms have high resolution and are capable of reliably detecting chromosomal structural abnormalities over $50 \mathrm{~kb}$ in size.

Pathogenic and likely pathogenic CNVs were detected in 84 subjects with NDDs (5 ASD and 79 ID/DD). Thus, the overall diagnostic yield was $20.9 \%$ in our cohort. However, it was significantly lower (3.3\%) in subjects with ASD, while remarkably higher (31.6\%) for subjects with ID/DD. We identified a de novo CNV in $14.9 \%$ of subjects with NDDs. We also found 20 ID/DD cases with more severe phenotypes who harbored two clinically relevant CNVs.

The diagnostic yield and de novo CNV rate of our cohort is comparable with some previous reports (Table 4). Our results are most consistent with Hu et al. ${ }^{20}$, who identified 127 cases carrying pathogenic CNVs in a cohort of 633 patients, obtaining a diagnostic yield of $20.06 \%$ for all NDD patients, $3.7 \%$ for isolated ASD, $18.07 \%$ for isolated ID/DD, and $34.90 \%$ for ID/DD with MCA. The size of CNVs identified in $\mathrm{Hu}$ et al. ranged from $223 \mathrm{~kb}$ to $102 \mathrm{Mb}$, and the de novo rate was $16.9 \%$. Lee et al. ${ }^{22}$ obtained a similar diagnostic yield of $32.2 \%$ in 177 patients with unexplained ID/DD. Fan et al. ${ }^{23}$ observed a yield of $28 \%$ in a mixed cohort of 710 Southern Chinese patients with NDDs. The highest yield was found in the subgroup of ID/DD with congenital heart defects (55\%), followed by ID/DD with facial dysmorphism (39\%), hypotonia (35\%), and microcephaly (34\%). Pinto et al. ${ }^{24}$ obtained a diagnostic yield of $3.4 \%$ in a cohort of 2446 subjects with ASD and a de novo rate of $4.7 \%$, but this was a strictly research cohort. This is comparable to our diagnostic yield of $2.6 \%$ in ASD subjects 
Table 4. Comparison of diagnostic yield CNVs with different studies.

\begin{tabular}{|c|c|c|c|c|c|c|}
\hline Total number of cases & CNV size $(\mathrm{kb})$ & De novo rate & \multicolumn{3}{|l|}{ Yield } & Source [reference no.] \\
\hline 402 & $56-95,100$ & $17.4 \%$ & $2.6 \%$ & $31.1 \%$ & $20.4 \%$ & This study \\
\hline 10,351 & NA & NA & $5.4 \%$ & $12.5 \%$ & $8.6 \%$ & Ho et al. $^{18}$ \\
\hline 633 & $223-102,000$ & $16.9 \%$ & $3.7 \%$ & $18.07-34.9 \%$ & $20.06 \%$ & Hu et al. $^{20}$ \\
\hline 2446 & $30-59,374$ & $4.7 \%$ & $3.4 \%$ & NA & $3.4 \%$ & Pinto et al. ${ }^{24}$ \\
\hline 10,619 & $30-5000$ & NA & NA & NA & $10.15 \%$ & Uddin et al. ${ }^{25}$ \\
\hline
\end{tabular}

and de novo rate $3.3 \%$. Ho et al. ${ }^{18}$ tested 10,351 NDDs cases and observed a yield of $5.4 \%$ for ASD/or combining with any other testing indications, which was also comparable to our ASD data. While their yield of $12.5 \%$ for subjects with ID/DD and $8.6 \%$ for all NDDs was lower than that observed in our study, it is comparable to some published data ${ }^{8,25}$. For example, Zarrei et al. ${ }^{8}$ obtained a yield of $10.5 \%$ in 1838 NDDs cases. A higher yield of $11.4 \%$ was observed in the ASD cases in this study. Uddin et al. ${ }^{25}$ also obtained a lower yield of $10.15 \%$ in a cohort of 10,619 subjects with NDDs. We suppose that the difference resulted from the constitution of cases with NDDs, which can include subjects with ASD, ADHD (attention deficit hyperactivity disorder), OCD (obsessive-compulsive disorder), and SCZ (schizophrenia) without many comorbid constructive defects. Our data further confirm that diagnostic yield is related positively with severities and comorbid conditions of NDDs, such as co-occurring facial dysmorphism and congenital heart diseases which could increase the yield markedly ${ }^{18,21}$.

The diagnostic yield of a cohort can be affected significantly by multiple factors that include but are not limited to: referring physician specialty, gender of patients, age of patient at testing, and referring indication (or combination of indications) for testing ${ }^{17}$. The specialties of the referring pediatricians who are responsible for selecting patients to undergo genetic testing constitutes the first bias to the diagnostic yield in a clinical setting ${ }^{17-19}$. In this study, a diagnostic yield of $31.5 \%$ was obtained for the patients with ID/DD presenting clear clinical features, some of whom suffered from more severe and complex phenotypes. These patients were mostly (96\%) diagnosed by senior developmental pediatricians or pediatric neurologists at the Pediatric Health Care Institute and Rehabilitation Center, and a few (4\%) by senior and experienced neonatologists at the Neonatology Department in our hospital. Their specialty and experiences in recognizing the patients' indications and severities is crucial to elevating the yield when referring them for genetic testing.

It is documented that the CNV burden differs between males and females in both NDD patients and the general population ${ }^{26-32}$. In our cohort, a higher diagnostic yield of pathogenic and likely pathogenic CNVs was observed in females (31.3\%) compared to males $(16.1 \%)$ when considering all NDDs, indicating a significant difference $\left(P=4.8 \times 10^{-4}\right)$. Similarly, in the ID/DD subgroup, a higher yield of $37.5 \%$ was observed in females compared to that of $27.2 \%$ in males, though this difference was not statistically significant. Jacquemont et al. ${ }^{26}$ investigated the molecular basis of the sex-based difference in a cohort of 15,585 probands with NDD, and found a significant increase in deleterious autosomal CNVs and single-nucleotide variants (SNVs) in female probands compared to males with NDDs. Jacquemont et al. also found that maternal transmission of deleterious CNVs and SNVs was observed more frequently in females than in males in an independent ASD cohort of 762 families. These data support the "female protective model", suggesting a higher "mutational burden" is required for females with NDDs to manifest clinical features. In our study, the CNV size in females was larger than that in males in the ID/DD subgroup (20.12 Mb $\pm 12.21 \mathrm{Mb}$ versus $2.45 \mathrm{Mb} \pm 2.07 \mathrm{Mb}$ ), demonstrating a significant difference between male and female patients $\left(P=1 \times 10^{-3}\right)$. This result is comparable to previous studies ${ }^{25,26}$. For example, Polyak et al. ${ }^{27}$ discovered that girls carried a higher burden of large CNVs and in both ASD and ID/DD cohorts. Han et al. $^{28}$ found a significant excess of large $(\geq 500 \mathrm{~kb})$, rare $(<1 \%)$ CNVs in females compared to males in both NDD cases and controls. Desachy et al. ${ }^{29}$ observed a similar phenomenon of large, rare CNVs in females in the population and ASD families, suggesting a female protective mechanism exists for deleterious CNVs that may go beyond NDDs phenotypes and contribute to decreased female fetal loss in the population. Roberts et al. ${ }^{32}$ observed a higher detection rate of abnormal CNVs in females (27\%) than in males (18\%). Roberts et al. also found that the average size of CNVs in ID/DD was much larger than that in ASD $(2.90 \pm 2.87 \mathrm{Mb}$ versus $966 \pm 1464 \mathrm{~kb})$. Consistently, in our cohort, the average size of CNVs in ID/DD cases was $4.14 \mathrm{Mb} \pm 3.77 \mathrm{Mb}$, which was much larger than that of ASD cases $(802.5 \mathrm{~kb} \pm$ $394.5 \mathrm{~kb}$ ). The difference is potentially related to the genetic cause of ID/DD and ASD, with smaller CNVs implicating a single gene in $A S D$ versus larger CNVs involving more than one gene in ID/DD.

It has been noticed that the age of patients also affects the detection rate of $\mathrm{CNVs}^{18,21}$. Xu et al. ${ }^{33}$ investigated CNVs identified in 434 patients with ASD and ID/DD. The yield for patients under 2 -year-old was $70 \%$, which was significantly higher than those over 5 years old. Most of the younger patients under 2-year-old suffered from comorbidity with severe medical problems, such as microcephaly, macrocephaly, hypotonia, and other systemic abnormalities like asphyxia of the newborn, malnutrition and anemia, which may have influenced the pediatricians to recommend genetic evaluation for these patients with CMA. Moreover, similar yields of $12 \%$ and $14.7 \%$ were obtained for both subgroups of ASD and ID/DD under 2-year-old in this study. In order to illustrate the impact of patients' age on the diagnostic yield, we stratified the diagnostic yields by age for both subgroups in our study, and found that the 2-5-year-old patients had the highest diagnostic yield of $3.5 \%(4 / 114)$ in ASD subgroup, while the highest yield of $40.3 \%$ (25/62) was observed in the 1-2-yearold cases in the ID/DD subgroup (Table 1). The 1-2-year-old ID/DD patients presented the most comorbidities for other abnormalities, such as cerebral palsy, facial dysmorphism, microcephalus, and epilepsy. The mean age of the subgroups in our study was significantly different $\left(P=1 \times 10^{-3}\right)$, being 4-year-1-month for ASD and 2-year-11-month for ID/DD, which may have contributed to the difference in yield between the two subgroups.

Aneuploidy is the gain or loss of an entire chromosome and is the leading genetic cause for developmental abnormality ${ }^{26}$. To better assess the advantages of CMA for identification of clinically 
relevant CNVs in $A S D$ and ID/DD with clear phenotypes in northern China, the patients with aneuploidy such as trisomy 21, 47XXY, $47 \mathrm{XYY}$ syndromes were excluded at first. In this study, 4 CNVs in ASD subgroup were associated with known chromosomal syndromes, including Kleefstra syndrome, AUTS2 syndrome, 15q24 microdeletion syndrome, and MECP2 duplication syndrome. In the ID/DD subgroup, $60 \mathrm{CNVs}$ occurred at loci associated with 37 known chromosomal syndromes. Recurrent pathogenic CNVs were most frequently detected at $7 q 11.23$, corresponding to the Williams-Beuren syndrome deletion which was observed in 11/ 251 (4.4\%) ID/DD patients. Other chromosomal syndromes observed in multiple patients with ID/DD included partial trisomy $9 p$ (duplication at chr9p24), 15q11q13 duplication syndrome, $18 q$ deletion syndrome ${ }^{34}$, Prader-Willi syndrome, 22q11 deletion syndrome (DiGeorge syndrome), 8p23.1 microdeletion syndrome, $22 q 11.2$ duplication syndrome, and MECP2 duplication syndrome.

It has been recommended that variants of uncertain significance be considered in the diagnostic yield of ID/DD, particularly ASD, as many CNVs of VUS will change to pathogenic CNVs following the accumulation of clinical evidence in databases ${ }^{31}$. By analyzing CNVs interpreted as VUS in this cohort, the diagnostic yield of ASD subgroup increased from 3.3\% (5/151) to 21.2\% (32/ 151). The 27 CNVs interpreted as VUS in the ASD subgroup included 17 duplications with size of $56 \mathrm{~kb}$ to $1.7 \mathrm{Mb}$ and 10 deletions with size of $74 \mathrm{~kb}$ to $1.2 \mathrm{Mb}$. These CNVs impacted some genes related to ASD/DD and were considered as new CNV candidates for association with ASD after analyzing data from databases and the literature. For example, a $141 \mathrm{~kb}$ duplication at 2q14.1 involving DPP10 was detected in a 3-year-old autistic boy. DPP10 has been reported to be related to synaptogenesis and ASD susceptibility in several studies of autism ${ }^{35-37}$. However, Mak et al. recently proposed that DPP10 duplication is likely a benign CNV polymorphism enriched in Southern Chinese with a population frequency of $\sim 1 \%$ by genotyping 258 Southern Chinese ASD patients $^{38}$. In DECIPHER, a CNV of the same size was reported in a patient with autistic behavior and mild global developmental delay. More samples, especially Chinese from northern China, are needed to clarify the genotype-phenotype relationship of this CNV. A second example is a $477 \mathrm{~kb}$ duplication at 2 q14.3 involving CNTNAP5 that was detected in a 2-year-4-month girl. Rare deletion of CNTNAP5 was suggested as a novel genetic factor that might confer ASD susceptibility ${ }^{39}$. However, duplications of CNTNAP5 have not been studied. Last, a $115 \mathrm{~kb}$ maternal duplication at 2q36.3 was identified in a 2-year-4-month female autistic patient with ASD, and overlaps TRIP12, which has been reported to be associated with $\mathrm{ASD}^{40}$. An $180 \mathrm{~kb}$ duplication at the $5^{\prime}$ portion of TRIP12 has been reported in an individual with macrocephaly ${ }^{41,42}$. Two additional duplications on 2q36.3 with similar sizes were found in two cases with ID and DD in the DECIPHER database.

In this study, we investigated the genetic etiology of a clinical cohort of NDD subjects who presented with ASD or ID/DD. The cohort was comprised of Han Chinese subjects from Shandong province, a northern region of China, who presented with typical phenotypes and were diagnosed by experienced pediatricians or pediatric neurologists. In our cohort, the patients with ID/DD were of a younger age than the patients with ASD. The high diagnostic yield observed in our cohort may have been influenced by the clinical experience of the referring pediatricians, the phenotypic severity of the cohort, gender, and age. Genome sequencing is expected to provide a higher yield on this clinical cohort ${ }^{43-45}$.

\section{METHODS}

\section{Ethics statement}

The study was approved by the Ethics Committee of Qilu Children's Hospital of Shandong University. Informed written consent was obtained from the patients' parents. The information of the patients' and their families was anonymized prior to genotyping and analysis.
All the procedures performed in the study were in accordance with the Declaration of Helsinki.

\section{Subjects}

A total of 410 probands with ASD or unexplained ID/DD with or without other congenital anomalies who were referred for genetic services in our institute from January 2014 to December 2018 were enrolled in this study. Their parents were also enrolled in the study. The cohort consisted of 282 male and 128 female patients (male:female ratio $=2.20$ ) with mean age of 2-years-11-months, including 151 unrelated ASD patients (127 males and 24 females, ratio $=5.29$ ) with mean age of 4-years-1-month (ranged from 1 -year-10-months to 8 -years-2-months) and 259 ID/DD patients (155 male and 104 female, ratio $=1.49$ ) with mean age of 1-year-10-month (ranged from 1 day to 10-year-3-month) (Supplementary Data 1). There were 40 fathers and 24 mothers who were unavailable for testing. The 151 ASD patients were diagnosed by experienced pediatric neurologists at the Pediatric Health Care Institute using the criteria defined in the Diagnostic and Statistical Manual of Mental Disorders, 5th Edition (DMS-5) (American Psychiatric Association, 2013), the Autism Diagnostic Observation Scale (ADOS-2, 2002), and confirmed with the Children Autism Rating Scale (CARS, score >30). The 259 ID/DD patients were diagnosed by experienced pediatric neurologists at the Pediatric Health Care Institute, Rehabilitation Center and Neurology Department in Qilu Children's Hospital of Shandong University according to the DMS-5 criteria, and the diagnosis was confirmed using the Gesell development scales with $\mathrm{DQ}<75$ and Wechsler Intelligence Scale for Children-Revised with IQ $<70$. The exclusion criteria for this study were: (1) organic diseases of the nervous system, such as cerebral palsy, chronic epilepsy, encephalitis, meningitis, severe brain injury, brain surgery; (2) severe systemic physical diseases, such as those of the heart, liver, kidney, endocrine, and circulation; (3) Schizophrenia and other mental disorders, such as attention deficit hyperactivity disorder (ADHD) and obsessive compulsive disorder (OCD); (4) abnormal organic acids in blood and urine screening tests.

All participants in this study were ethnically and geographically homogenous Han Chinese recruited from Shandong province. Five subjects with highly suspected trisomy 21 syndrome were analyzed by G-banding karyotyping based on recommendation of the clinicians, and the remaining 405 patients were analyzed by CMA using the Affymetrix CytoScan HD array or Affymetrix Human Genome-Wide SNP 6.0 array.

\section{Genotyping analysis}

Genomic DNA was extracted from peripheral blood samples of the probands and their parents using TIANamp Blood Genomic DNA Purification Kit (TIANGEN, Beijing, China) following the manufacturer's instructions. Potential RNA contamination was removed by RNaseA (TIANGEN, Beijing, China). The DNA was quantified using the NanoDrop ND-1000 spectrophotometer (Thermo Fisher, Waltham, MA, USA). The genomic DNA was genotyped using the Affymetrix Human Genome-Wide SNP Array 6.0 or Affymetrix CytoScan HD Arrays (Affymetrix, Santa Clara, Calif., USA). DNA digestion, ligation, fragmentation, labeling, hybridization, staining and scanning were performed following the manufacturer's protocols (Affymetrix, Santa Clara, CA).

\section{Data analysis and CNV evaluation}

The data were analyzed with Command Console 3.1 (Affymetrix, Santa Clara, CA) or Chromosome Analysis Suite (ChAS) version 3.1.0.15 (Affymetrix, Santa Clara, Calif., USA). Data quality was evaluated with contrast quality control $(C Q C)$. The default $C Q C$ threshold $(\geq 0.4)$ was used for analyzing each sample. Samples with a CQC $<0.4$ were excluded from the study. The QC call rates of all the samples were greater than $96 \%$. The reporting threshold was set at $50 \mathrm{~kb}$ (markers $\geq 20$ ) for deletions and duplications.

To evaluate the pathogenic associations of CNVs, the Database of Genomic Variants (DGV, http://projects.tcag.ca/variation), CAG database (CAGdb, http://www.cagdb.org), University of California Santa Cruz Genome Browser (UCSC, http://genome.ucsc.edu), Online Mendelian Inheritance in Man (OMIM, http://www.omim.org), DECIPHER database (http://decipher.sanger.ac.uk), ISCA (https://www.iscaconsortium.org), and PubMed (http://www.ncbi.nlm.nih.gov/pubmed) were used. The control data of 1679 non-ASD Chinese subjects from multiple sources were used to distinguish rare copy number variations in our cohort. The control data included 919 samples from Singapore database ${ }^{46}, 103$ samples from the HapMap project ${ }^{47}, 451$ samples from Lu et al. ${ }^{48}$, and 206 parents from 
Gazzellone et al. ${ }^{49}$. The frequency of prioritized CNVs was computed against the aforementioned controls. CNVs with $>50 \%$ reciprocal overlap were deemed identical ${ }^{50}$. Rare CNVs were defined as those not being present in more than $1 \%$ of 1679 ethnically-matched non-ASD control samples. We further restricted rare CNVs to those not being present in more than $1 \%$ of subjects in the Database of Genomic Variants (DGV) ${ }^{51}$. We analyzed CNVs overlapping $<70 \%$ of their total length with segmental duplications and repeat-rich loci of the human genome. All CNVs were classified as pathogenic, likely pathogenic, variants of uncertain significance (VUS), likely benign, and benign according to the American College of Medical Genetics and Genomics guidelines ${ }^{52}$. In brief, CNVs were considered as pathogenic if they were documented as clinically significant in multiple peer-reviewed publications, or large CNVs unreported in the literature but overlapped a smaller interval with established clinical significance; CNVs were regarded as likely pathogenic if they were described in a single case report but with well-defined breakpoints and phenotype associated with NDDs, or involved a gene with a very compelling function-related and specific to NDDs. Other classes of CNVs were considered variants of uncertain clinical significance (VUS), likely benign or benign CNVs. Pathogenic, likely pathogenic, and VUS CNVs were considered to potentially affect gene function associated with the phenotypes of ID/DD or ASD in the study and were further validated by MLPA/qPCR.

\section{Multiplex ligation-dependent probe amplification (MLPA)/ quantitative PCR (qPCR)}

MS-MLPA was performed using SALSA MLPA kits ME028 and ME030 (MRC Holland, Amsterdam, Netherlands) to identify PWS/AS, and Silver-Russell syndrome, separately, according to the manufacturer's instructions. The data were analyzed using Coffalyser software. qPCR with SYBR Green chemistry were utilized to verify the potentially clinically relevant CNVs in the cases and their parents. The primer sets for qPCR were designed to target different fragments within variant regions using an online primer designing tool-Primer 3 (http://primer3.ut.ee/) - and were synthesized by Shanghai Invitrogen Biotechnology Company (Shanghai, China) (Supplementary Data 5 and 6). Assays were carried out in accordance with manufacturer recommendations on the 7500 Fast Real-Time PCR system (Applied Biosystems, Foster City, California). The copy number variations were determined based on the ratio of target region copies to reference gene $(G A P D H)$ copies in samples. Both male and female genomic DNA samples from unaffected pooled samples stored in our laboratory were used simultaneously as male and female control samples. Each qPCR was carried out in triplicate with the SYBR Premix Ex Taq II PCR reagent kit (TakaRa Bio, Dalian, China) following the manufacturer's protocol.

\section{Statistical analysis}

Statistical analysis was performed with SPSS 16.0 software. Two-sided Fisher's test was used to test significance of CNVs in different groups. Results were considered statistically significant when the $P$ value was $<0.05$ and the confidence interval was $95 \%$.

\section{Reporting summary}

Further information on research design is available in the Nature Research Reporting Summary linked to this article.

\section{DATA AVAILABILITY}

CNVs data has been deposited at the European Genome-phenome Archive (EGA), which is hosted by the EBI and the CRG, under accession number EGAS00001005763. Further information about EGA can be found on http://ega-archive.org.

Received: 21 December 2019; Accepted: 9 November 2021; Published online: 12 January 2022

\section{REFERENCES}

1. Curry, C. J. et al. Evaluation of mental retardation: recommendations of a consensus conference. Am. Coll. Med. Genet. Am. J. Med. Genet. 72, 468-77 (1997).

2. Boyle, C. A. et al. Trends in the prevalence of developmental disabilities in US children, 1997-2008. Pediatrics 127, 1034-1042 (2011).
3. Ceylan, A. C. et al. Importance and usage of chromosomal microarray analysis in diagnosing intellectual disability, global developmental delay, and autism; and discovering new loci for these disorders. Mol. Cytogenet 11, 54 (2018).

4. Stessman, H. A. et al. Targeted sequencing identifies 91 neurodevelopmentaldisorder risk genes with autism and developmental-disability biases. Nat. Genet. 49, 515-526 (2017)

5. Autism Spectrum Disorders Working Group of The Psychiatric Genomics Consortium. Meta-analysis of GWAS of over 16,000 individuals with autism spectrum disorder highlights a novel locus at 10q24.32 and a significant overlap with schizophrenia. Mol. Autism 8, 21 (2017).

6. Woodbury-Smith, M. \& Scherer, S. W. Progress in the genetics of autism spectrum disorder. Dev. Med. Child Neurol. 60, 445-451 (2018).

7. Miller, D. T. et al. Consensus statement: Chromosomal microarray is a first-tier clinical diagnostic test for individuals with developmental disabilities or congenital anomalies. Am. J. Hum. Genet. 86, 749-764 (2010).

8. Zarrei, M. et al. A large data resource of genomic copy number variation across neurodevelopmental disorders. NPJ Genom. Med. 4, 26 (2019).

9. Moeschler, J. B. \& Shevell, M. Committee on Genetics. Committee on genetics comprehensive evaluation of the child with intellectual disability or global developmental delays. Pediatrics 134, e903-e918 (2014).

10. Schaefer, G. B. \& Mendelsohn, N. J., Professional Practice and Guidelines Committee. Clinical genetics evaluation in identifying the etiology of autism spectrum disorders: 2013 guideline revisions. Genet. Med. 15, 399-407 (2013).

11. Shevell, M. et al. Practice parameter: evaluation of the child with global developmental delay: report of the Quality Standards Subcommittee of the American Academy of Neurology and The Practice Committee of the Child Neurology Society. Neurology 60, 367-380 (2003).

12. Elia, J. et al. Genome-wide copy number variation study associates metabotropic glutamate receptor gene networks with attention deficit hyperactivity disorder. Nat. Genet. 44, 78-84 (2011).

13. Kandaswamy, R. et al. Allelic association, DNA resequencing and copy number variation at the metabotropic glutamate receptor GRM7 gene locus in bipolar disorder. Am. J. Med. Genet. B Neuropsychiatr. Genet. 165B, 365-372 (2014)

14. Kearney, H. M. et al. American College of Medical Genetics standards and guidelines for interpretation and reporting of postnatal constitutional copy number variants. Genet. Med. 13, 680-685 (2011).

15. Fan, Y. et al. Rare Copy Number Variations in a Chinese Cohort of Autism Spectrum Disorder. Front. Genet. 9, 665-380 (2018).

16. Sharma, P. et al. Application of chromosomal microarrays in the evaluation of intellectual disability/global developmental delay patients-A study from a tertiary care genetic centre in India. Gene 590, 109-119 (2016).

17. Oikonomakis, V. et al. Recurrent copy number variations as risk factors for autism spectrum disorders: analysis of the clinical implications. Clin. Genet. 89, 708-718 (2016).

18. Ho, K. S. et al. Chromosomal microarray analysis of consecutive individuals withautism spectrum disorders using an ultra-high resolution chromosomal microarray optimized for neurodevelopmental disorders. Int. J. Mol. Sci. 17 (2016).

19. Battaglia, A. et al. Confirmation of chromosomal microarray as a first-tier clinical diagnostic test for individuals with developmental delay, intellectual disability, autism spectrum disorders and dysmorphic features. Eur. J. Paediatr. Neurol. 17, 589-599 (2013).

20. Hu, T. et al. Chromosomal Aberrations in Pediatric Patients with Developmental Delay/Intellectual Disability: A Single-Center Clinical Investigation. Biomed. Res. Int. 2019, 1-16 (2019).

21. Micleaa, D. et al. Genomic study via chromosomal microarray analysis in a group of Romanian patients with obesity and developmental disability/intellectual disability. J. Pediatr. Endocrinol. Metab. 32, 667-674 (2019).

22. Lee, C. L. et al. Array-CGH increased the diagnostic rate of developmental delay or intellectual disability in Taiwan. Pediatr. Neonatol. 60, 453-460 (2019).

23. Fan, Y. et al. Chromosomal microarray analysis in developmental delay and intellectual disability withcomorbid conditions. BMC Med. Genomics 11, 49 (2018).

24. Pinto, D. et al. Convergence of genes and cellular pathways dysregulated in autism spectrum disorders. Am J Hum Genet 94, 677-694 (2014).

25 . Uddin, M. et al. Indexing effects of copy number variation on genes involved in developmental delay. Sci Rep 6, 28663 (2016).

26. Jacquemont, S. et al. A higher mutational burden in females supports a "female protective model" in neurodevelopmental disorders. Am. J. Hum. Genet. 94, 415-425 (2014).

27. Polyak, A. et al. An assessment of sex bias in neurodevelopmental disorders. Genome Med. 7, 94 (2015).

28. Han, J. et al. Gender differences in CNV burden do not confound schizophrenia CNV associations. Sci. Rep. 6, 25986 (2016). 
29. Desachy, G. et al. Increased female autosomal burden of rare copy number variants in human populations and in autism families. Mol. Psychiatry 20, 170-175 (2015).

30. Ruderfer, D. M. et al. Patterns of genic intolerance of rare copy number variation in 59,898 human exomes. Nat. Genet. 48, 1107-1111 (2016).

31. Männik, K. et al. Copy number variations and cognitive phenotypes in unselected populations. JAMA 313, 2044-2054 (2015).

32. Roberts, J. L. et al. Chromosomal microarray analysis of consecutive individuals with autism spectrum disorders or learning disability presenting for genetic services. Gene 535, 70-78 (2014).

33. $\mathrm{Xu}, \mathrm{M}$. et al. Clinical application of chromosome microarray analysis in Han Chinese children with neurodevelopmental disorders. Neurosci. Bull. 34, 981-991 (2018).

34. Treff, N. R., Su, J., Taylor, D. \& Scott, R. T. Jr. Telomere DNA deficiency is associated with development of human embryonic aneuploidy. PLoS Genet 7, e1002161 (2011).

35. Marshall, C. R. et al. Structural variation of chromosomes in autism spectrum disorder. Am. J. Hum. Genet. 82, 477-488 (2008).

36. Girirajan, S. et al. Refinement and discovery of new hotspots of copy-number variation associated with autism spectrum disorder. Am. J. Hum. Genet 92, 221-237 (2013).

37. Lin, M. et al. Heat shock alters the expression of schizophrenia and autism candidate genes in an induced pluripotent stem cell model of the human telencephalon. PLoS One 9, e94968 (2014).

38. Mak, A. S. L. et al. Use of clinical chromosomal microarray in Chinese patients with autism spectrum disorder-implications of a copy number variation involving DPP10. Mol. Autism 8, 31 (2017).

39. Pagnamenta, A. T. et al. Characterization of a family with rare deletions in CNTNAP5 and DOCK4 suggests novel risk loci for autism and dyslexia. Biol. Psychiatry 68, 320-328 (2010).

40. O'Roak, B. J. et al. Recurrent de novo mutations implicate novel genes underlying simplex autism risk. Nat. Commun. 5, 5595 (2014).

41. Bramswig, N. C. et al. Identification of new TRIP12 variants and detailed clinical evaluation of individuals with non-syndromic intellectual disability with or without autism. Hum. Genet. 136, 179-192 (2017).

42. Zhang, J. et al. Haploinsufficiency of the E3 ubiquitin-protein ligase gene TRIP12 causes intellectual disability with or without autism spectrum disorders, speech delay, and dysmorphic features. Hum. Genet. 136, 377-386 (2017).

43. Stavropoulos, D. J. et al. Whole-genome sequencing expands diagnostic utility and improves clinical management in paediatric medicine. NPJ Genom. Med. 1, 15012 (2016).

44. Clark, M. M. et al. Meta-analysis of the diagnostic and clinical utility of genome and exome sequencing and chromosomal microarray in children with suspected genetic diseases. NPJ Genom. Med. 3, 16 (2018).

45. Srivastava, S. et al. Meta-analysis and multidisciplinary consensus statement: exome sequencing is a first-tier clinical diagnostic test for individuals with neurodevelopmental disorders. Genet Med. 21, 2413-2421 (2019).

46. Teo, Y. Y. et al. Singapore Genome Variation Project: a haplotype map of three Southeast Asian populations. Genome Res. 19, 2154-2162 (2009).

47. International HapMap 3 Consortium, Altshuler, D. M. et al. Integrating common and rare genetic variation in diverse human populations. Nature 467, 52-58 (2010).

48. Lu, J. et al. Assessing genome-wide copy number variation in the Han Chinese population. J. Med. Genet. 54, 685-692 (2017)

49. Gazzellone, M. J. et al. Copy number variation in Han Chinese individuals with autism spectrum disord er. J. Neurodev. Disord. 6, 34 (2014).

50. Zarrei, M., MacDonald, J. R., Merico, D. \& Scherer, S. W. A copy number variation map of the human genome. Nat. Rev. Genet. 16, 172-183 (2015).

51. MacDonald, J. R., Ziman, R., Yuen, R. K., Feuk, L. \& Scherer, S. W. The database of genomic variants: a curated collection of structural variation in the human genome. Nucleic Acids Res. 42, D986-992 (2014).
52. South, S. T. et al. ACMG Standards and Guidelines for constiturional cytogenomic microarray analysis, including postnatal and prenatal applications: revision 2013. Genet Med. 15, 901-909 (2013).

\section{ACKNOWLEDGEMENTS}

The project is financially supported by Science and Technology Project of Jinan (201602194) and National Natural Science Foundation of China (81671362). The authors are grateful to the patients and their families for their contribution to the project. We would like to thank The Centre for Applied Genomics at the Hospital for Sick Children and the University of Toronto McLaughlin Centre. S.W.S. holds the Norhbridge Chair in Paediatric Research, a joint Hospital-University Chair between the University of Toronto, The Hospital for Sick Children, and the SickKids Foundation.

\section{AUTHOR CONTRIBUTIONS}

This study was conceived and designed by Y.L., S.W.S., and Z.G. The experiments were conducted by Y.L., X.Y., Y.Li., H.Z., and Y.W. Data analyzed and interpreted by Y.L., Y.Lv., M.Z., E.J.H., and S.W.S. R.D., D.Z., F.S., Y.Y., and Z.G. contributed clinical diagnosis of the patients. The paper was written by Y.L. and revised by M.Z., E.J.H., and S.W.S. Y.L.v. was considered as a co-first author.

\section{COMPETING INTERESTS}

S.W.S. is on the Scientific Advisory Committees of Deep Genomics, Population Bio and serves as a Highly Cited Academic Advisor for the King Abdulaziz University. The remaining authors declare no competing interests. S.W.S. is Editor-in-Chief of npj Genomic Medicine.

\section{ADDITIONAL INFORMATION}

Supplementary information The online version contains supplementary material available at https://doi.org/10.1038/s41525-021-00271-z.

Correspondence and requests for materials should be addressed to Stephen W. Scherer or Zhongtao Gai.

Reprints and permission information is available at http://www.nature.com/ reprints

Publisher's note Springer Nature remains neutral with regard to jurisdictional claims in published maps and institutional affiliations.

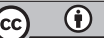

Open Access This article is licensed under a Creative Commons Attribution 4.0 International License, which permits use, sharing, adaptation, distribution and reproduction in any medium or format, as long as you give appropriate credit to the original author(s) and the source, provide a link to the Creative Commons license, and indicate if changes were made. The images or other third party material in this article are included in the article's Creative Commons license, unless indicated otherwise in a credit line to the material. If material is not included in the article's Creative Commons license and your intended use is not permitted by statutory regulation or exceeds the permitted use, you will need to obtain permission directly from the copyright holder. To view a copy of this license, visit http://creativecommons. org/licenses/by/4.0/.

(c) The Author(s) 2022 